\title{
MicroRNA-210 knockdown contributes to apoptosis caused by oxygen glucose deprivation in PC12 cells
}

\author{
JIE QIU, XIAO-YU ZHOU, XIAO-GUANG ZHOU, YONG LI, RUI CHENG and HAI-YING LIU \\ Department of Newborn Infants, Nanjing Children's Hospital of Nanjing Medical University, \\ Nanjing, Jiangsu 210008, P.R. China
}

Received January 9, 2014; Accepted August 8, 2014

DOI: $10.3892 / \mathrm{mmr} .2014 .2651$

\begin{abstract}
It was previously demonstrated that microRNA-210 (miR-210) exhibited neuroprotective effects in a murine model of hypoxic-ischemic encephalopathy via inhibition of apoptosis. The aim of the present study was to further elucidate the effect of miR-210 on apoptosis in PC12 cells following transfection with miR-210 inhibitors and exposure to oxygen glucose deprivation (OGD). The expression levels of miR-210 were identified using reverse transcription-quantitative polymerase chain reaction analysis. Apoptosis was investigated using Annexin V-fluorescein isothiocyanate assays. Apoptosis-related protein expression levels were studied with western blot analysis. The results showed that the expression levels of miR-210 were upregulated in PC12 cells following a 4-h exposure to OGD, relative to those in normoxic control cells. miR-210 knockdown increased cell apoptosis by inducing caspase activity and regulating the balance between $\mathrm{Bcl}-2$ and Bax levels. The present study demonstrated that miR-210 knockdown induced cell apoptosis using an ex vivo model of ischemic hypoxia ( $\mathrm{IH})$. Knockdown of miR-210 represents a potential novel therapeutic approach to combat neonatal IH.
\end{abstract}

\section{Introduction}

Hypoxic-ischemic encephalopathy (HIE) is the primary cause of cerebral damage and long-term neurological sequelae, including cerebral palsy and mental retardation (1), in the perinatal period in term and preterm infants (2). However, no specific and successful neuroprotective strategies currently exist. Brain protection in newborn infants remains a challenging priority and represents a medical requirement that has yet to be met.

Recently, microRNAs (miRNAs), a large conserved family of noncoding short RNAs, have been found to be novel

Correspondence to: Dr Jie Qiu, Department of Newborn Infants, Nanjing Children's Hospital of Nanjing Medical University, 72 Guangzhou Road, Nanjing, Jiangsu 210008, P.R. China

E-mail: jieqiu.nj@hotmail.com

Key words: microRNA-210, hypoxic-ischemic encephalopathy, oxygen glucose deprivation, apoptosis therapeutic tools for the treatment of ischemic hypoxia (IH). Hypoxia and insufficient blood flow to the brain, known as brain ischemia, lead to poor oxygen supply and consequently the death of brain tissue, with a wide range of pathophysiological outcomes. miRNAs have previously been reported to be induced by oxygen deprivation. In particular, microRNA-210 (miR-210), which is activated by the transcriptional factor hypoxia inducible factor- $1 \alpha(\mathrm{HIF}-1 \alpha)$ (3), is a unique miR that is evolutionarily conserved and ubiquitously expressed in a number of hypoxic cell and tissue types (4-10). HIF-1 $\alpha$ is an important factor in the molecular mechanisms of oxygen deprivation (11-16) and the development of injured and normal brains. Hence, we hypothesized that miR-210 may be involved in HIE, even though its exact role remains to be determined. Its association with HIE may establish miR-210 as a potential therapeutic target.

During normal brain development, redundant neurons are removed via apoptosis; this is an important physiological process that ensures the formation of appropriate neural networks. However, following IH, this apoptotic component becomes detrimental, leading to excessive neuronal loss. Therefore, in a previous study (17), the effect of miR-210 on the IH-induced apoptosis of neurons was investigated in an ex vivo model of IH (18-20), through the use of PC12 rat pheochromocytoma cells combined with oxygen glucose deprivation (OGD). The results of the study demonstrated that miR-210 overexpression suppressed cell apoptosis by inhibiting caspase activity and regulating the balance between the expression levels of Bcl-2 and Bax. The aim of the present study was to further elucidate the effect of miR-210 on apoptosis in PC12 cells via the silencing of its expression.

\section{Materials and methods}

Cell culture. PC12 rat pheochromocytoma cells were obtained from the American Tissue Culture Collection (Rockville, MD, USA) and cultured in Dulbecco's modified Eagle's medium (DMEM) supplemented with $10 \% \mathrm{v} / \mathrm{v}$ horse serum (HS), $5 \% \mathrm{v} / \mathrm{v}$ fetal bovine serum (FBS) and appropriate antibiotics in a humidified chamber $\left(5 \% \mathrm{CO}_{2}\right.$ and $\left.37^{\circ} \mathrm{C}\right)$, all of which were purchased from Invitrogen Life Technologies (Carlsbad, CA, USA).

miRNA transfection. An hsa-miR-210 inhibitor (anti-miR210) or anti-miR-negative control \#1 (Ambion, Austin, TX, USA) 
(50 $\mu$ l) was diluted in OptiMEM I (Invitrogen, Carlsbad, CA, USA) to a concentration of $100 \mathrm{nM}$. Subsequently, $50 \mu \mathrm{l}$ of the solution was mixed with $50 \mu 1$ Lipofectamine 2000 (Invitrogen) in OptiMEM I (25X dilution), incubated at room temperature for $20 \mathrm{~min}$ and then added to each well of a 24-well plate $(100 \mu \mathrm{l})$. PC1 2 cells $\left(400 \mu 1\right.$, concentration $6.25 \times 10^{5}$ cells $\left./ \mathrm{ml}\right)$ were added to each well. The transfection mixture was incubated $\left(5 \% \mathrm{CO}_{2}\right.$ and $37^{\circ} \mathrm{C}$ ) for $24 \mathrm{~h}$ and the cells were either used immediately in assays or the media was replaced (500 $\mu \mathrm{l}$ DMEM/10\% v/v $\mathrm{HS} / 5 \% \mathrm{v} / \mathrm{v} \mathrm{FBS}$ ) and the mixture incubated further.

Oxygen glucose deprivation (OGD). A mixture of 95\% nitrogen and $5 \% \mathrm{CO}_{2}$ was passed through glucose-free DMEM to deoxygenate it. The DMEM was used to wash the PC12 cells once and then to maintain them prior to use. The cells were then placed in a modular incubation chamber (Billups-Rothenberg, Del Mar, CA, USA) and flushed with $95 \%$ nitrogen $/ 5 \% \mathrm{CO}_{2}$ for $4 \mathrm{~min}$ at a flow rate of $10 \mathrm{l} / \mathrm{min}$. The chamber was sealed and stored in an incubator for $4 \mathrm{~h}$ at $37^{\circ} \mathrm{C}$. Control cells were washed with glucose-containing DMEM and incubated in a normoxic incubator for $4 \mathrm{~h}$, as previously described (17).

miRNA reverse transcription-quantitative polymerase chain reaction ( $R T-q P C R)$. Total RNA was prepared using TRIzol $^{\circledR}$ reagent (Invitrogen). miRNA was purified using the mirVana ${ }^{\mathrm{TM}}$ kit (Applied Biosystems, Foster City, CA, USA), according to the manufacturer's instructions. Using specific miR-210 and an endogenous control U6 stem-loop primer (Applied Biosystems, Foster City, CA, USA), reverse transcription was performed using the TaqMan ${ }^{\circledR}$ MicroRNA Reverse Transcription kit (Applied Biosystems) according to the manufacturer's instructions. Total RNA (10 ng) was reverse transcribed to cDNA using the following reagents: $1 \mathrm{mM}$ dNTPs (with dTTP), $1 \mu 1$ reverse transcriptase $(50 \mathrm{U} / \mu \mathrm{l})$ and RNase inhibitor $(4 \mathrm{U})$ in the presence of specific miR-210 or U6 stem-loop reverse transcriptase primers in a 15- $\mu 1$ system buffered with RT buffer and diethylpyrocarbonate (DEPC) water. The RT thermal cycle program was as follows: $16^{\circ} \mathrm{C}$ for $30 \mathrm{~min}, 42^{\circ} \mathrm{C}$ for $30 \mathrm{~min}$ and $85^{\circ} \mathrm{C}$ for $5 \mathrm{~min}$. The resulting cDNA was stored at $-20^{\circ} \mathrm{C}$. The qPCR step was performed using a 7900HT Fast Real-Time PCR system with a TaqMan ${ }^{\circledR}$ MicroRNA Assay kit (Applied Biosystems). The total reaction volume was $20 \mu 1$ and contained the following components: miR-210 or U6 RT reaction product $(1.33 \mu \mathrm{l})$, $1 \mu \mathrm{l}$ 20X TaqMan ${ }^{\circledR}$ MicroRNA assay (for miR-210 or U6), $10 \mu \mathrm{l} 2 \mathrm{X}$ TaqMan Universal PCR Master mix and $7.67 \mu 1$ DEPC water (Sigma-Aldrich, St. Louis, MO, USA). qPCR was then performed on the 96-well plate using the following protocol: $95^{\circ} \mathrm{C}$ for $10 \mathrm{~min}$, followed by 43 cycles of $95^{\circ} \mathrm{C}$ for $15 \mathrm{sec}$ and $60^{\circ} \mathrm{C}$ for $1 \mathrm{~min}$. The relative miR-210 level was normalized to the expression of the endogenous control U6 for each sample, calculated using the $2^{-\Delta \Delta \mathrm{Ct}}$ method. Experiments were performed in triplicate.

Evaluation of apoptotic index. Cells were harvested using trypsin/ethylenediaminetetraacetic acid (EDTA) (Sigma-Aldrich), washed with phosphate-buffered saline (PBS; Sigma-Aldrich), resuspended in $100 \mu \mathrm{l}$ binding buffer (BioVision, Milpitas, CA, USA) and stained with $5 \mu 1$ Annexin V-fluorescein isothiocyanate (FITC) and

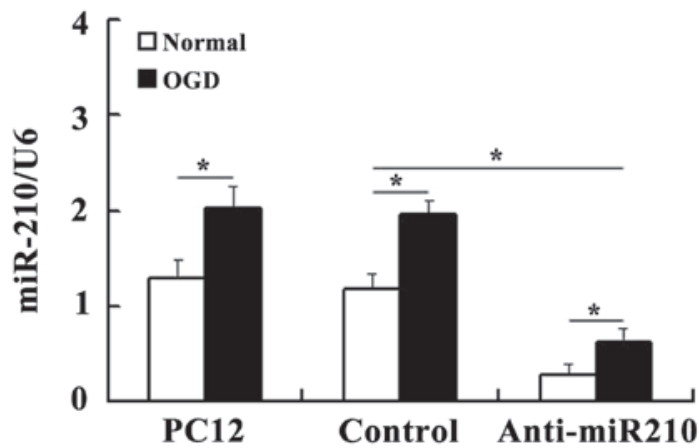

Figure 1. MicroRNA (miR)-210 expression was upregulated in cells following a 4-h period of oxygen glucose deprivation (OGD) compared with cells under normoxic conditions (normal). miR-210 expression was downregulated in the cells transfected with anti-miR210 compared with the PC12 and negative control vector cells. Data are presented as the mean \pm standard deviation of three independent experiments performed in triplicate $\left({ }^{*} \mathrm{P}<0.05\right)$.

$1 \mu \mathrm{l}$ propidium iodide (PI) at room temperature for $15 \mathrm{~min}$ (Biovision). The fluorescence of FITC and PI was analyzed using flow cytometry (Becton Dickinson, Mountain View, CA) following the addition of $400 \mu \mathrm{l}$ binding buffer.

Western blot analysis. Cells were washed with ice-cold PBS and lysed in protein lysis buffer $(50 \mathrm{mmol} / \mathrm{l} \mathrm{Tris}, 150 \mathrm{mmol} / \mathrm{l} \mathrm{NaCl}$, $10 \mathrm{mmol} / \mathrm{l}$ EDTA, $1 \%$ Triton X-100, $200 \mathrm{mmol} / \mathrm{l} \mathrm{NaF}$ and $4 \mathrm{mmol} / \mathrm{l}$ sodium orthovanadate-containing protease inhibitors, $\mathrm{pH}$ 7.5) for $1 \mathrm{~h}$ on ice. Proteins were quantified using a bicinchoninic acid (BCA) Protein Assay kit (Pierce, Rockford, IL, USA) according to the manufacturer's instructions. Following separation by $10 \%$ sodium dodecyl sulfate-polyacrylamide gel electrophoresis, the proteins ( $20 \mu \mathrm{g} / \mathrm{lane})$ were electrophoretically transferred onto a nitrocellulose membrane (Whatman, London, UK), which was blocked with non-fat dry milk in buffer. The membrane was incubated with mouse monoclonal antibodies against caspase-3, caspase-9, Bax and Bcl-2 (Santa Cruz Biotechnology, Inc., Santa Cruz, CA, USA) and goat anti-mouse $\mathrm{IgG}$ secondary antibodies conjugated with horseradish peroxidase (Santa Cruz Biotechnology, Inc.) according to the manufacturer's instructions. Thereafter the proteins were visualized with an electrochemiluminescence detection system (GE Healthcare Bio-Sciences, Uppsala, Sweden) and analyzed using Quantity One Analysis software (Bio-Rad Laboratories, Hercules, CA, USA). $\beta$-actin (Cell Signaling Technology, Inc., Beverly, MA, USA) was used as protein loading control.

Statistical analysis. All data are expressed as the mean \pm the standard error of the mean. Statistical analysis was performed using the paired Student's t-test of the SPSS statistical software package v10.0 (SPSS, Inc., Chicago, IL, USA). P $<0.05$ was considered to indicate a statistically significant difference.

\section{Results}

OGD significantly increases miR-210 expression levels. The expression levels of miR-210 were measured using RT-qPCR analysis. U6 was used as the endogenous control as it was the most stably expressed miR across all subjects in the control 


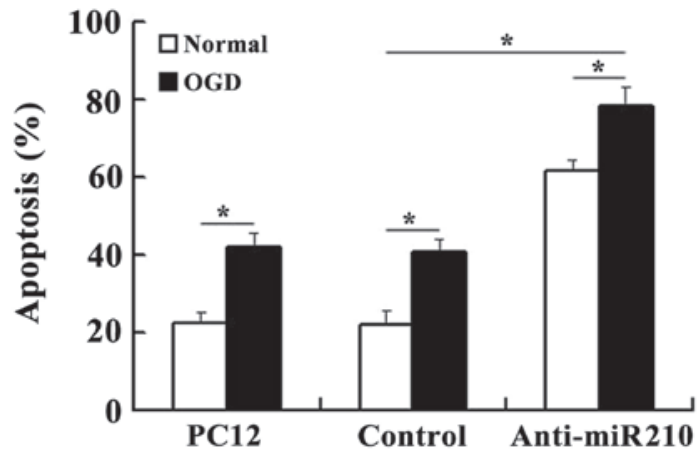

Figure 2. Effect of microRNA (miR)-210 on cell apoptosis. Apoptosis was upregulated in cells following a 4-h period of oxygen glucose deprivation (OGD) compared with cells under normoxic conditions (normal). Apoptosis was upregulated in the cells transfected with anti-miR210 compared with the PC12 cells or cells transfected with the negative control vector. Values shown are the mean \pm standard deviation of three independent experiments performed in triplicate $\left({ }^{*} \mathrm{P}<0.05\right)$.

and experimental groups (data not shown). Expression of miR-210 was markedly downregulated in cells transfected with anti-miR210 compared with that in the U6 controls, which confirmed that the miR-210 knockdown cells had been prepared successfully. Consistent with previous results (17), RT-qPCR revealed that miR-210 expression was significantly induced in PC12 cells under hypoxic conditions (Fig. 1).

miR-210 knockdown increases the levels of cell apoptosis. As shown in Fig. 2, compared with the control cells that were not deprived of glucose and maintained under normoxic conditions, the cells subjected to OGD showed a higher level of cell death. However, miR-210 knockdown increased the levels of apoptosis observed in cells, particularly following subjection to OGD.

miR-210 alters the protein expression levels of caspase-3, caspase-9, Bax and Bcl-2. The effects of miR-210 on the expression levels of apoptosis-related proteins were assessed. Western blot analysis demonstrated that the protein levels of Bax, caspase- 3 and caspase- 9 were increased in the miR-210 knockdown cells compared with those observed in the controls. By contrast, anti-apoptotic Bcl-2 expression levels changed in an almost inverse manner (Fig. 3).

\section{Discussion}

miRNAs are small noncoding RNAs that silence the expression of target genes through either mRNA degradation or the suppression of transcription procedures. While several studies have demonstrated the roles of specific miRNAs in neuronal differentiation, neurogenesis, neural cell specification and neurodevelopmental function (21-23), no studies are available on the importance of miRNAs in HIE to the best of our knowledge. Recently, a specific group of hypoxia-regulated miRNAs that are under the regulation of HIF-1 $\alpha$ were identified. miR-210, a direct transcriptional target of HIF-1 $\alpha$, is one of the most hypoxia-sensitive miRNAs and has a number of pleiotropic effects. miR-210 has been mechanistically linked to the control of a wide range of cellular responses known to influence normal developmental physiology, as well as a

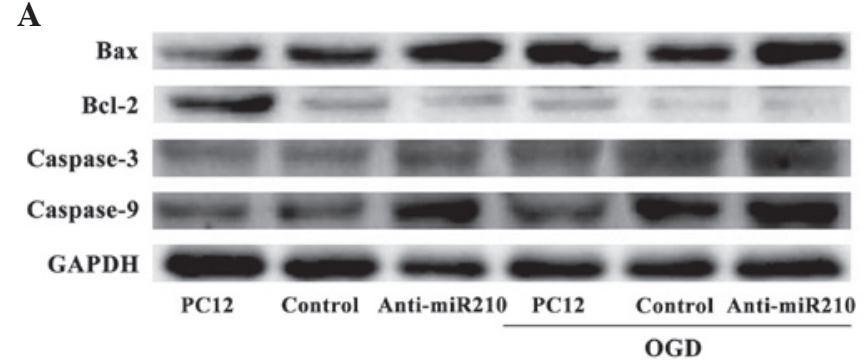

B
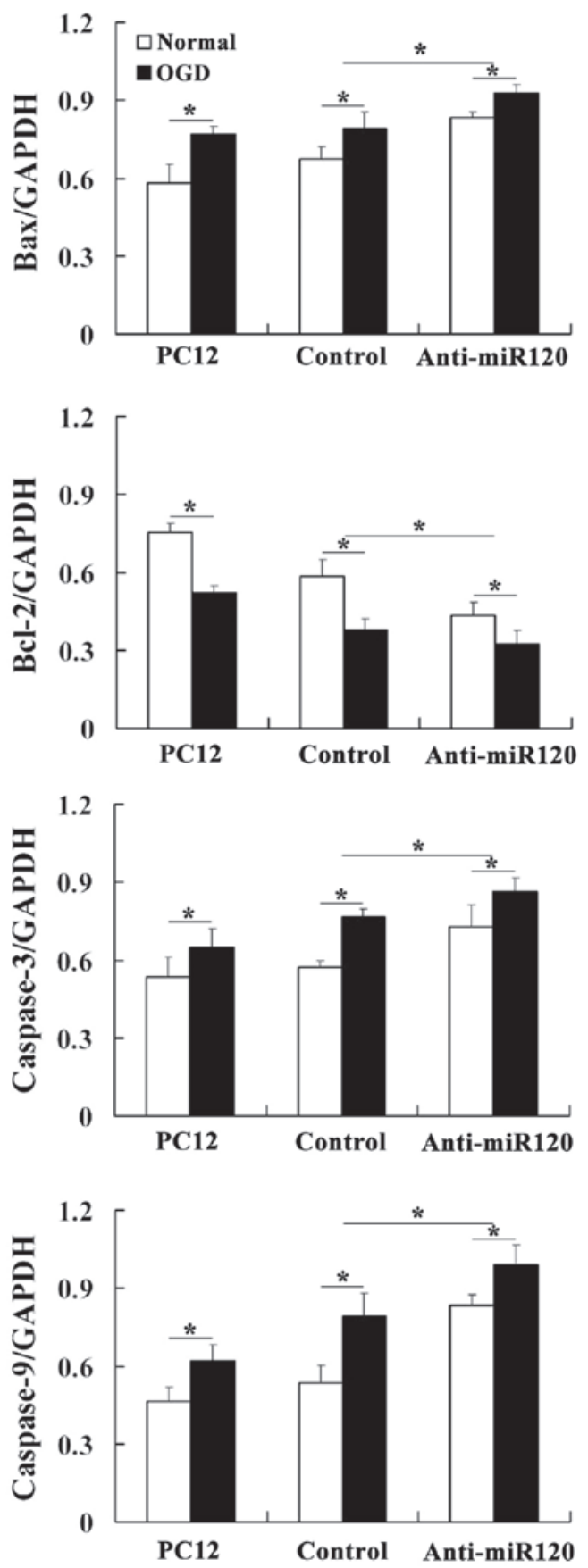

Figure 3. Effects of microRNA (miR)-210 on the protein expression levels of caspase-3, caspase-9, Bax and Bcl-2 in normal cells and those subjected to oxygen glucose deprivation (OGD). (A) Western blot analysis shows that in the miR-210 knockdown cells (anti-miR210), the protein expression of caspase-3, caspase-9 and Bax protein increased, whilst that of anti-apoptotic Bcl-2 decreased compared with that in the PC12 or control cells. (B) Quantification of results. Density values shown are the mean \pm standard deviation of three independent experiments performed in triplicate $\left({ }^{*} \mathrm{P}<0.05\right)$. 
number of hypoxia-dependent disease states, including tissue ischemia, inflammation and tumorigenesis.

A previous study demonstrated that miR-210 has neuroprotective effects through inhibiting apoptosis in a murine model of HIE (24). Furthermore, an OGD model was used to investigate the link between miR-210 and IH injury in PC12 cells through the overexpression of miR-210 (17). To further elucidate the effect of miR-210 on apoptosis in PC12 cells following exposure to OGD, the present study used anti-miRNA transfection to downregulate the expression of miR-210.

A previous study has shown that miR-210 expression is upregulated in PC12 cells following a 4-h exposure to OGD (17). Consistently, the current study also demonstrated that OGD upregulates the expression of miR-210 in both miR-210 knockdown and control cells. The induction of miR-210 by hypoxia is consistent with studies involving other types of cell, including embryo kidney cells, endothelial cells, breast carcinoma cells, colonic adenocarcinoma cells and ovarian epithelial cells $(8,25,26)$. Thus far, in all published studies, miR-210 is an miRNA that has been consistently observed to be upregulated in normal and transformed hypoxic cells to the best of our knowledge.

miRNAs are involved in a diverse range of biological processes, including development, cell growth, apoptosis and hematopoiesis. During normal brain development, redundant neurons are removed via apoptosis; this is an important physiologic process to ensure the formation of appropriate neural networks. However, following IH, this apoptotic component becomes detrimental, leading to excessive neuronal loss. Previous studies have revealed that miR-210 treatment within the first $4 \mathrm{~h}$ post-OGD prevents cell apoptosis (17). The present study showed that anti-miR210 transfection induced apoptosis in both normoxic and hypoxic conditions, which demonstrates that miR-210 modulates the cellular apoptotic response to OGD. In support of this finding, the results revealed that miR-210 inhibition upregulates expression levels of Bax, caspase-3 and caspase-9; while downregulating Bcl-2 expression. Thus, it may be hypothesized that the miR-210 inhibition-dependent upregulation of Bax, caspase- 3 and caspase- 9 expression and downregulation of Bcl-2 expression may contribute to the modulation of the apoptotic response to IH.

miRNA-based therapeutics are set to become the one of the most significant commercial hotspots in the health care market (27-31). Although there are a number of challenges for miRNAs as therapeutic targets, including delivery, potential off-target effects and safety, the strategy of manipulating miRNAs in vivo to regulate disease-related processes is already becoming a feasible therapeutic approach for the future (32), due to the small size of miRNAs and their high rate of transduction in eukaryotic cells. Furthermore, the feasibility of miRNAs as therapeutics is supported by ongoing clinical trials (33). In the present study, we hypothesize that miR-210 may have a significant role in regulating apoptosis following IH injury and may lead to a novel therapy for the treatment of HIE.

In conclusion, the present study demonstrated that the knockdown of miR-210, which is a highly upregulated miRNA induced by OGD, contributes to OGD-induced neuronal apoptosis through the induction of caspase activity and the regulation of the balance between Bcl-2 and Bax expression levels. Further investigation of the regulation, targets and physiological and/or pathogenic effects of miR-210 in IH should be anticipated in the future.

\section{Acknowledgements}

This study was supported by grants from the Science and Technology Development Project of Nanjing, China (no.: 201001090), the Medical Science and Technology Development Project of the Nanjing Health Bureau, China (no.: YKK10046) and the Nanjing Sanitation Engineering of Young Talents during the $12^{\text {th }}$ Five-Year Plan Period.

\section{References}

1. Tioseco JA, Aly H, Essers J, Patel K and El-Mohandes AA: Male sex and intraventricular hemorrhage. Pediatr Crit Care Med 7: 40-44, 2006.

2. du Plessis AJ and Volpe JJ: Perinatal brain injury in the preterm and term newborn. Curr Opin Neurol 15: 151-157, 2002.

3. Ivan M, Harris AL, Martelli F and Kulshreshtha R: Hypoxia response and microRNAs: no longer two separate worlds. J Cell Mol Med 12: 1426-1431, 2008.

4. Chan SY and Loscalzo J: MicroRNA-210: a unique and pleiotropic hypoxamir. Cell Cycle 9: 1072-1083, 2010.

5. Chan SY, Zhang YY, Hemann C, Mahoney CE, Zweier JL and Loscalzo J: MicroRNA-210 controls mitochondrial metabolism during hypoxia by repressing the iron-sulfur cluster assembly proteins ISCU1/2. Cell Metab 10: 273-284, 2009.

6. Chen Z, Li Y, Zhang H, Huang $\mathrm{P}$ and Luthra R: Hypoxia-regulated microRNA-210 modulates mitochondrial function and decreases ISCU and COX10 expression. Oncogene 29: 4362-4368, 2010.

7. Favaro E, Ramachandran A, McCormick R, Gee H, et al: MicroRNA-210 regulates mitochondrial free radical response to hypoxia and krebs cycle in cancer cells by targeting iron sulfur cluster protein ISCU. PloS One 5: e10345, 2010.

8. Huang X, Ding L, Bennewith KL, Tong RT, et al: Hypoxia-inducible mir-210 regulates normoxic gene expression involved in tumor initiation. Mol Cell 35: 856-867, 2009.

9. Kushibiki T: Photodynamic therapy induces microRNA-210 and -296 expression in HeLa cells. J Biophotonics 3: 368-372, 2010.

10. Pulkkinen K, Malm T, Turunen M, Koistinaho J and Yla-Herttualä S: Hypoxia induces microRNA miR-210 in vitro and in vivo ephrin-A3 and neuronal pentraxin 1 are potentially regulated by miR-210. FEBS Lett 582: 2397-2401, 2008.

11. Bacon AL and Harris AL: Hypoxia-inducible factors and hypoxic cell death in tumour physiology. Ann Med 36: 530-539, 2004

12. Gordan JD and Simon MC: Hypoxia-inducible factors: central regulators of the tumor phenotype. Curr Opin Genet Dev 17: 71-77, 2007.

13. Gruber M and Simon MC: Hypoxia-inducible factors, hypoxia, and tumor angiogenesis. Curr Opin Hematol 13: 169-174, 2006.

14. Harris AL: Hypoxia - a key regulatory factor in tumour growth. Nat Rev Cancer 2: 38-47, 2002.

15. Kim JW, Tchernyshyov I, Semenza GL and Dang CV: HIF-1-mediated expression of pyruvate dehydrogenase kinase: a metabolic switch required for cellular adaptation to hypoxia. Cell Metab 3: 177-185, 2006.

16. Koumenis C: ER stress, hypoxia tolerance and tumor progression. Curr Mol Med 6: 55-69, 2006.

17. Qiu J, Zhou XY, Zhou XG, Cheng R, Liu HY and Li Y: Neuroprotective effects of microRNA-210 against oxygen-glucose deprivation through inhibition of apoptosis in PC12 cells. Mol Med Rep 7: 1955-1959, 2013.

18. Tabakman R, Lazarovici P and Kohen R: Neuroprotective effects of carnosine and homocarnosine on pheochromocytoma PC12 cells exposed to ischemia. Journal Neurosci Res 68: 463-469, 2002.

19. Guo G and Bhat NR: p38alpha MAP kinase mediates hypoxia-induced motor neuron cell death: a potential target of minocycline's neuroprotective action. Neurochem Res 32: 2160-2166, 2007. 
20. Tabakman R, Jiang H, Schaefer E, Levine RA and Lazarovici P: Nerve growth factor pretreatment attenuates oxygen and glucose deprivation-induced c-Jun amino-terminal kinase 1 and stress-activated kinases p38alpha and p38beta activation and confers neuroprotection in the pheochromocytoma PC12 model. J Mol Neurosci 22: 237-250, 2004.

21. Kim J, Krichevsky A, Grad Y, Hayes GD, Kosik KS, Church GM and Ruvkun G: Identification of many microRNAs that copurify with polyribosomes in mammalian neurons. Proc Nat Acad Sci USA 101: 360-365, 2004.

22. Kosik KS and Krichevsky AM: The elegance of the microRNAs: A neuronal perspective. Neuron 47: 779-782, 2005.

23. Schratt GM, Tuebing F, Nigh EA, Kane CG, Sabatini ME, Kiebler M and Greenberg ME: A brain-specific microRNA regulates dendritic spine development. Nature 439: 283-289, 2006.

24. Qiu J, Zhou XY, Zhou XG, Cheng R, Liu HY and Li Y: Neuroprotective effects of microRNA-210 on hypoxicischemic encephalopathy. Biomed Res Int 2013: 350419, 2013.

25. Fasanaro P, D'Alessandra Y, Di Stefano V, Melchionna R, et al: MicroRNA-210 modulates endothelial cell response to hypoxia and inhibits the receptor tyrosine kinase ligand Ephrin-A3. J Biol Chem 283: 15878-15883, 2008.
26. Giannakakis A, Sandaltzopoulos R, Greshock J, Liang S, Huang J, Hasegawa K, Li C, O'Brien-Jenkins A, Katsaros D, Weber BL, Simon C, Coukos G and Zhang L: miR-210 links hypoxia with cell cycle regulation and is deleted in human epithelial ovarian cancer. Cancer Biol Ther 7: 255-264, 2008.

27. van Rooij E, Liu N and Olson EN. MicroRNAs flex their muscles. Trends Genet 24: 159-166, 2008.

28. Love TM, Moffett HF and Novina CD: Not miR-ly small RNAs: big potential for microRNAs in therapy. J Allergy Clin Immunol 121: 309-319, 2008.

29. Weiler J, Hunziker J and Hall J: Anti-miRNA oligonucleotides (AMOs): ammunition to target miRNAs implicated in human disease? Gene Ther 13: 496-502, 2006.

30. Ørom UA, Kauppinen S and Lund AH: LNAmodified oligonucleotides mediate specific inhibition of microRNA function. Gene 372: 137-141, 2006

31. Zhang B and Farwell MA: microRNAs: a new emerging class of players for disease diagnostics and gene therapy. J Cell Mol Med 12: 3-21,2008.

32. Liu NK and Xu XM: MicroRNA in central nervous system trauma and degenerative disorders. Physiol Genomics 43: 571-580, 2011.

33. Zhang C: Novel functions for small RNA molecules. Curr Opin Mol Ther 11: 641-651, 2009. 